\title{
Fosfatagem e Calagem no desenvolvimento do capim mombaça
}

\author{
Phosphating and Liming in the development of mombasa grass \\ Fosfatado y Encalado en el desarrollo de la hierba mombaça
}

Recebido: 11/03/2021 | Revisado: 20/03/2021 | Aceito: 14/10/2021 | Publicado: 16/10/2021

\author{
Kátia Cristina Gavilak da Costa \\ ORCID: https://orcid.org/0000-0002-5702-2580 \\ Universidade Estadual de Mato Grosso do Sul, Brasil \\ E-mail: katiagavilak@gmail.com \\ Francisco Eduardo Torres \\ ORCID: https://orcid.org/0000-0002-6114-0096 \\ Universidade Estadual de Mato Grosso do Sul, Brasil \\ E-mail: feduardo@uems.br \\ Marcos Jefferson Kraeski \\ ORCID: https://orcid.org/0000-0002-1026-8057 \\ Universidade Estadual de Mato Grosso do Sul, Brasil \\ E-mail: marcos_kraeski@hotmail.com \\ Angelita dos Santos Zanuncio \\ ORCID: https://orcid.org/0000-0002-7191-8498 \\ Universidade Estadual de Mato Grosso do Sul, Brasil \\ E-mail: angelitazanuncio@hotmail.com \\ Gleiciane de Lima Benteo \\ ORCID: https://orcid.org/0000-0002-8111-2682 \\ Universidade Estadual de Mato Grosso do Sul, Brasil \\ E-mail: gleicianebenteo@ hotmail.com
}

\begin{abstract}
Resumo
O objetivo deste trabalho foi verificar a interação entre a calagem e a fosfatagem em diferentes profundidades no crescimento do Panicum maximum cv. Mombaça a fim de obter melhor manejo desses produtos de forma a ter uma recomendação adequada. $\mathrm{O}$ experimento foi conduzido no campo agrostológico situado na área experimental da Universidade Estadual de Mato Grosso do Sul - Unidade Universitária de Aquidauana. O delineamento foi inteiramente casualizado, com três repetições, disposto em esquema fatorial 4 x 5 , com 4 saturações de base (sem calagem, $\mathrm{V}=40 \%, 50 \%$ e $60 \%$ ) e 5 profundidades de fosfato natural de Bonito (sem fosfato, fosfato na superfície, $10 \mathrm{~cm}, 20 \mathrm{~cm}$ e $30 \mathrm{~cm}$ ), totalizando 54 unidades experimentais. O solo utilizado para o experimento possuía $23,30 \%$ de saturação de bases. Da parte aérea avaliou-se o comprimento das folhas, largura das folhas e número de perfilhos, além de produção de massa seca total (MST), massa de colmos (COL), massa de folhas (FOL), massa de material morto $(\mathrm{MM})$ e a relação folha:colmo $(\mathrm{F} / \mathrm{C})$. Em relação às raízes foi observado comprimento de raiz (RAI) e massa seca total. A correção da saturação de bases para $40 \%$ proporciona os melhores resultados de CF, LF e NP, independente da profundidade de aplicação de fósforo. A profundidade de aplicação de fósforo não altera as variáveis CR, MM, RAI, FOL, COL e F/C. A profundidade de aplicação de fósforo, na ausência de calagem, interferiu no CF, LF e NP. A adubação fosfatada é recomendada para independente da profundidade de aplicação.
\end{abstract}

Palavras-chave: Adubação fosfatada; Fosfato natural; Panicum maximum cv. mombaça.

\begin{abstract}
The work's aim was to verify the interaction between cathe and phosphating at different depths in the mombasa grass growth in order to obtain better management of these products in order to have an adequate recommendation. The experiment was conducted in the agrostological field located in the experimental area of the State University of Mato Grosso do Sul - University Unit of Aquidauana. The design was completely randomized, with three replications, arranged in a factorial scheme $4 \times 5$, with 4 base saturations ( $\mathrm{V}=40 \%, 50 \%, 60 \%$ and without plowing) and 5 depths of Bonito natural phosphate (without phosphate, phosphate on the surface, 10, 20 and $30 \mathrm{~cm}$ ), totaling 54 experimental units. The soil used for the experiment had 23,30\% base saturation. From the aerial part, leaf length, leaf width and number of byums were evaluated, in addition to total dry mass production (MST), stem mass (COL), leaf mass (FOL), dead material mass (MM) and leaf:stem (F/C) ratio. In relation to the roots, root length (RAI) and total dry mass were observed. The correction of base saturation to $40 \%$ provides the best results of CF, LF and NP, regardless of the depth of phosphorus application. The depth of phosphorus application doesn't alter the variables CR, MM, RAI, FOL, COL and F/C. The depth of phosphorus application, in the absence of cathe, interfered in the CF, LF and NP. Phosphate fertilization is recommended for regardless of depth of application.
\end{abstract}

Keywords: Phosphated fertilization; Natural phosphate; Panicum maximum cv. mombasa. 


\begin{abstract}
Resumen
El objetivo de este trabajo era verificar la interacción entre el cátodo y la fosfatación a diferentes profundidades en el crecimiento máximo de $\mathrm{cv}$ de panicum mombaça con el fin de obtener una mejor gestión de estos productos con el fin de tener una recomendación adecuada. El experimento se llevó a cabo en el campo agrostológico ubicado en el área experimental de la Universidad Estatal de Mato Grosso do Sul - Unidad Universitaria de Aquidauana. El diseño fue completamente aleatorizado, con tres replicaciones, dispuestas en un esquema factorial 4 x 5 , con 4 saturaciones básicas ( $\sin$ caté, $\mathrm{V}=40 \%, 50 \%$ y $60 \%$ ) y 5 profundidades de fosfato natural bonito (sin fosfato, fosfato en la superficie, $10 \mathrm{~cm}, 20 \mathrm{~cm}$ y $30 \mathrm{~cm}$ ), con un total de 54 unidades experimentales. El suelo utilizado para el experimento tenía un 23,30\% de saturación de base. Desde la parte aérea, se evaluaron la longitud de la hoja, la anchura de la hoja y el número de byums, además de la producción total de masa seca (MST), la masa del tallo (COL), la masa de las hojas (FOL), la masa de material muerto (MM) y la relación hoja:vástago (F/C). En relación con las raíces, se observaron la longitud de la raíz (RAI) y la masa seca total. La corrección de la saturación base al $40 \%$ proporciona los mejores resultados de CF, LF y NP, independientemente de la profundidad de la aplicación de fósforo. La profundidad de aplicación del fósforo no altera las variables CR, MM, RAI, FOL, COL y F/C. La profundidad de aplicación del fósforo, en ausencia de cátodo, interfirió en el CF, LF y NP. Se recomienda la fertilización de fosfato para independientemente de la profundidad de aplicación.
\end{abstract}

Palabras clave: Fertilización de fosfato; Fosfato natural; Panicum maximum cv. mombaça.

\title{
1. Introdução
}

A atividade pecuária no Brasil tem como base uma das maiores áreas de pastagem do planeta, utilizada para a criação dos maiores rebanhos bovinos do mundo. Essa característica de ter a grande maioria do seu rebanho bovino criado a pasto reduz custos de produção e eleva a qualidade da carne brasileira, tornando-a uma das mais competitivas do planeta (Dias-Filho, 2016).

A maioria dos solos brasileiros são comumente ácidos, o que pode causar problemas para plantios comerciais. Além da alta saturação de alumínio, que pode ter efeitos tóxicos para as plantas, os solos ácidos normalmente contêm baixo teor de cálcio e magnésio trocáveis, cátions de grande importância para o desenvolvimento radicular. Uma das alternativas para minimizar esse problema é a correção da acidez através da prática da calagem (Goedert et al, 1987).

A calagem dos solos ácidos aumenta a assimilabilidade e a captação por parte dos vegetais, no que toca a certos elementos como molibdênio, fósforo, cálcio e magnésio. Ao mesmo tempo a calagem reduz de maneira drástica as concentrações de ferro, alumínio e manganês que, sob condições de elevada acidez, poderão atingir quantidades tóxicas. Os efeitos da calagem ocorrem principalmente na estimulação dos organismos heterotróficos de finalidade geral. O estimulo favorece, não só a formação do húmus, como auxilia também na eliminação de certos produtos orgânicos intermediários que poderão mostrar-se tóxicos aos vegetais superiores (Rossa, 2006).

As recomendações para se elevar a saturação de base por meio de calagem devem ser feitas de acordo com a exigência das plantas, sendo a saturação de base de 30 a 35\% para gramíneas pouco exigentes (Andropogon gayanus, Brachiaria decumbens, Brachiaria humidicola e Brachiaria ruziziensis), de 40 a $45 \%$ para plantas exigentes (Brachiaria brizantha e Panicum maximum cv. vencedor e centenário) e 50 a $60 \%$ para forragens muito exigentes (Panicum maximum cv. colonião, tobiatã, tanzânia e mombaça; e Cynodon spp) (Souza, et al, 2001).

O capim Mombaça tem grande repercussão dentro da pecuária brasileira, tendo em vista a elevada produção forrageira, boa aceitabilidade e bom valor nutritivo. Para isso, é necessário um manejo eficiente, que seja capaz de dar à forrageira todas as condições necessárias para expressar o seu potencial (Araújo et al, 2019).

Outro fator importante que deve ser considerado é que a maioria dos solos ácidos apresenta baixos teores de P-Lábil, devido a grande adsorção do nutriente por partículas do solo, limitando assim a eficiência da adubação fosfatada. Assim, a calagem é um passo fundamental para otimizar a utilização pelas plantas de nutrientes que estão sendo adicionados via adubação (Ramos et al, 1993). 
O fósforo atua na fotossíntese, na respiração, no armazenamento e na transferência de energia, na divisão celular, no crescimento das células e em vários outros processos nas plantas. As plantas absorvem a maior parte do seu fósforo como íon ortofosfato primário $\left(\mathrm{H}_{2} \mathrm{PO}_{4}{ }^{-}\right)$, mas, pequenas quantidades de íon ortofosfato secundário (HPO42-) são também absorvidas (Lopes, 1998).

Nesse contexto, objetivou-se nesse trabalho verificar a interação entre a calagem e a fosfatagem em diferentes profundidades no crescimento do capim mombaça a fim de obter melhor manejo desses produtos de forma a ter uma recomendação adequada a cultura.

\section{Metodologia}

O experimento foi instalado no campo Agrostológico, localizado na área experimental da Universidade Estadual de Mato Grosso do Sul - Unidade Universitária de Aquidauana (UEMS/UUA), setor de fitotecnia.

A Universidade está localizada na região de transição entre os biomas Cerrado e Pantanal, situado no município de

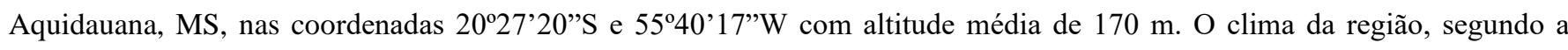
classificação descrita por Köppen-Geiger é do tipo Aw (Tropical de Savana) com precipitação média anual de $1.200 \mathrm{~mm}$ e temperatura média de $26,2^{\circ} \mathrm{C}$.

$\mathrm{O}$ delineamento experimental utilizado foi inteiramente casualizado, com três repetições, disposto em esquema fatorial 4 x 5, na qual os fatores foram constituídos de 4 saturações de base [V $=40 \%, \mathrm{~V}=50 \%, \mathrm{~V}=60 \%$ e sem calagem (T)] - fator quantitativo, e 5 situações de fosfato natural - fator qualitativo [sem fosfato (T), fosfato colocado em superfície (S), colocado a $10 \mathrm{~cm}$ de profundidade (10), a $20 \mathrm{~cm}$ de profundidade (20) e a $30 \mathrm{~cm}$ de profundidade (30)], totalizando 54 unidades experimentais.

As unidades experimentais foram constituídas de canos de PVC com $50 \mathrm{~cm}$ de comprimento e $20 \mathrm{~cm}$ de diâmetro, com capacidade para $20 \mathrm{~kg}$ de solo. O solo utilizado no experimento, classificado como Latossolo vermelho distroférrico (EMBRAPA, 2018), possuía baixo teor de fósforo (Tabela 1), ou seja, com possibilidade para elevar o seu teor. Este solo foi coletado numa área de pastagem antiga sem adubação nos últimos 15 anos.

Em agosto de 2020 foi feita a incubação do solo para o preenchimento das unidades experimentais, para tanto, procedeu-se a calagem para a elevação da saturação de bases conforme dados da análise de solo (Tabela 1) e também para alcançar os níveis recomendados para forrageiras. Sendo assim, foi utilizado calcário dolomítico (PRNT=100\%, $\mathrm{CaO}=28 \%$, $\mathrm{MgO}=21,5 \%, \mathrm{PN}=103 \%$ ), na quantidade para atingir as saturações de bases necessárias (Raij et al, 1997).

Tabela 1. Características químicas e granulométricas do solo (UEMS - Aquidauana/MS 2019).

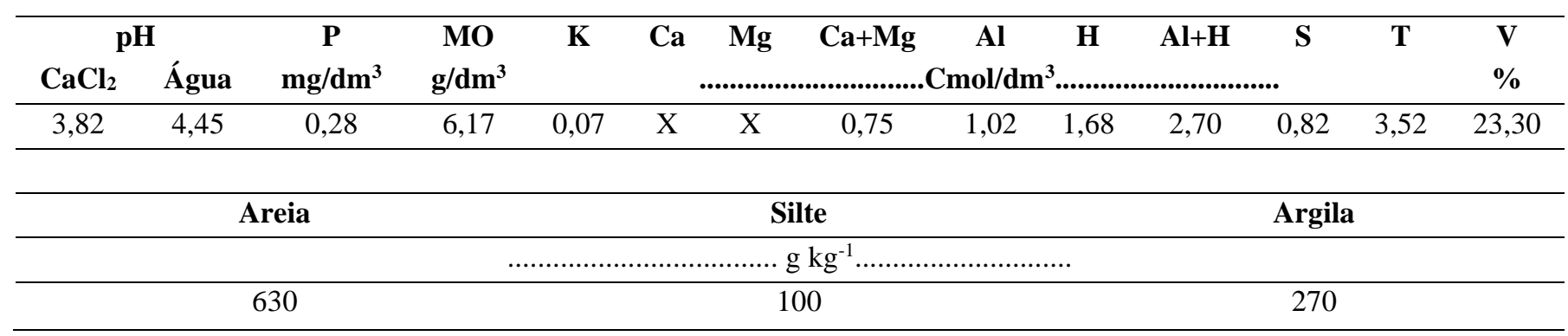

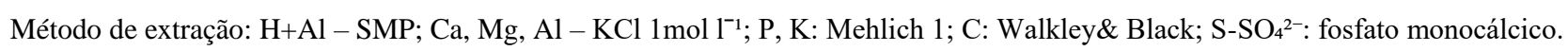

Fonte: Laboratório de Análise de Solo e Consultoria Ltda. Campo Grande - MS.

Para elevar as saturações de bases foram utilizadas as seguintes quantidades de calcário: 580 kg.ha-1 para saturação 40 $\%, 930 \mathrm{~kg} \cdot \mathrm{ha}^{-1}$ para saturação $50 \%$ e, $1.290 \mathrm{~kg} \cdot \mathrm{ha}^{-1}$ para saturação $60 \%$. Em seguida, esses solos foram incubados por um 
período de 35 dias (Duda \& Salviano, 2007). A incubação foi feita em galpão de alvenaria coberto, no qual a quantidade de solo necessária para o preenchimento dos tubos, de acordo com os tratamentos, sendo o calcário colocado sobre o piso formando três volumes de acordo com a saturação de base. A cada dois dias, esse solo foi revolvido usando enxada, para a ação do calcário.

Após o período de incubação do solo as unidades experimentais foram montadas com tubos de PVC, dispostos de forma aleatória, um ao lado do outro, num espaço dentro do Campo Agrostológico, que foi cercado com réguas de madeira, para impedir a entrada de animais.

O enchimento dos tubos foi feito com solo (após a incubação), colocado dentro do tubo na profundidade de acordo com os tratamentos e adicionado o fósforo, na quantidade de 2,40 g para elevar o P para $12 \mathrm{mg} / \mathrm{dm}^{3}$ (RAIJ et al., 1997). Essa quantidade de fósforo foi distribuída de maneira homogênea sobre o solo com o Fosfato Reativo de Bonito (15,7 \% de P), nas profundidades de $30 \mathrm{~cm}, 20 \mathrm{~cm}, 10 \mathrm{~cm}$ e na superfície conforme os tratamentos, e em seguida o tubo foi completado com solo novamente.

A semeadura com Panicum maximum cv. Mombaça aconteceu no dia 28 de setembro de 2020 e foi feita de maneira manual na quantidade em torno de 15 sementes por tubo. Aos 20 dias após a emergência das plantas (DAE) realizou-se o desbaste, no qual permaneceram cinco plantas/tratamento. Nesse mesmo dia foi feita a aplicação de Nitrogênio na forma de ureia, na quantidade de $100 \mathrm{~kg} \cdot \mathrm{ha}^{-1}$, correspondente a $2 \mathrm{~g}$ por tubo, na adubação de formação, além de $2 \mathrm{~g} \mathrm{KCl}\left(58 \%\right.$ de $\mathrm{K}_{2} \mathrm{O}$ ), na quantidade de $60 \mathrm{~kg} \cdot \mathrm{ha}^{-1}$ com quantidades idênticas para todos os tratamentos. As aplicações desses minerais aconteceram aos 20 e aos 30 DAE.

Durante todo o experimento as unidades experimentais foram mantidas a campo, com os tubos sem isolamento e a umidade mantida por meio de regas controladas para repor a água evapotranspirada.

Aos 41 DAE foi realizada observação para analisar o desenvolvimento da parte aérea, observando-se:

- Comprimento da folha (CF) em cm;

- Largura da folha (LF) em cm;

- Número de perfilhos (NP), contando manualmente cada perfilho.

Nesse mesmo dia as plantas foram retiradas dos tubos e separadas parte aérea de raiz. As plantas foram cortadas rente ao solo e, da parte aérea separada em lâminas foliares, colmos + bainhas e material morto, e acondicionadas em sacos de papel. Essas embalagens foram colocadas em estufa de circulação de ar forçado a $65^{\circ} \mathrm{C}$ por $72 \mathrm{~h}$, para obtenção da matéria seca, sendo feita no Laboratório de Química da UEMS.

Desse material foram observados a produção de massa seca total (MST), massa de colmos (COL), massa de folhas (FOL), massa de material morto (MM) e a relação folha:colmo (F/C).

Imediatamente à retirada da parte aérea, o solo foi retirado do tubo, lavado com água corrente, retirada o excesso de umidade com papel toalha, acondicionado em sacos de papel, e submetido à secagem similarmente à parte aérea para observar massa de raiz (RAI).

Para obtenção dos valores de comprimento de raiz $\mathrm{CR}$, as raízes foram sobrepostas sobre uma malha e obtida uma imagem digital seguindo a metodologia proposta por Tennant (1975), sendo utilizada a seguinte equação:

$$
C R=\left(\frac{\pi}{4}\right) \times N \times G
$$

Sendo:

- $\mathrm{CR}$ o comprimento de raiz em $\mathrm{cm}$,

- $\pi$ uma constante matemática,

- $\mathrm{N}$ o número de intersecções e 
- G a unidade da malha em $\mathrm{cm}$.

Em uma bandeja plástica foi fixada no fundo uma folha de papel milimetrado $(1 \mathrm{~cm} \times 1 \mathrm{~cm})$ e adicionado água na quantidade para encobrir o papel milimetrado. Em seguida, foi retirada subamostra da raiz $(0,2 \mathrm{~g})$ e distribuída na bandeja; fotografou-se e procedeu-se a contagem das intersecções para encontrar o valor de $\mathrm{N}$ para cada tratamento.

Depois de atendidos os pressupostos da análise de variância (normalidade e homocedasticidade), havendo a necessidade de retirada de outliers, prosseguiu-se com a mesma. O fator profundidade de fósforo foi considerado qualitativo e se observada diferença significativa entre as médias prosseguiu-se com o teste de Tukey. $\mathrm{O}$ fator calagem foi considerado quantitativo e se constatada diferença significativa prosseguiu-se com a análise de regressão. Foram ajustados modelos lineares com ajuste simples, quadrático e cúbico, e observados os ajustes pelo coeficiente de determinação e a representatividade do modelo ao fenômeno, escolhendo-se o melhor modelo que descreve os dados. As análises estatísticas foram realizadas utilizando os pacotes Expedes (Expedes: fat2. rbd; Ferreira et al, 2014) e ggplot2 (Wickham, 2010) no software R (ver. 3.5.2 Team, 2018).

\section{Resultados e Discussão}

A profundidade de aplicação do fosfato reativo de Bonito não influenciou as variáveis comprimento de raiz (CR), matéria morta (MM), massa de raiz (RAI), massa de folha (FOL), massa de colmo (COL) e na relação folha/colmo(F/C) (Tabela 2). Esses resultados estão diretamente relacionados a absorção de fósforo pelo sistema radicular, visto que o desenvolvimento deste em todos os tratamentos foi maior que as profundidades de aplicação do fósforo no solo, possibilitando assim um bom desenvolvimento das plantas.

Tabela 2. Resultados de comprimento de raiz (CR), matéria morta (MM), massa de raiz (RAI), massa de folha (FOL), massa de colmo (COL) e na relação folha/colmo (F/C), em função das doses de calcário e da profundidade de aplicação de fósforo.

\begin{tabular}{|c|c|c|c|c|c|c|c|}
\hline & & $\mathrm{CR}(\mathrm{cm})$ & MM (g) & RAI $(g)^{1}$ & FOL $(g)^{2}$ & $\operatorname{COL}(\mathrm{g})^{2}$ & $\mathrm{~F} / \mathrm{C}^{3}$ \\
\hline \multirow{5}{*}{$\begin{array}{l}\text { PROF } \\
\text { (P) }\end{array}$} & Sem & 66,65 & 0,09 & 1,03 & 0,76 & 0,62 & 1,40 \\
\hline & Sup & 61,87 & 0,13 & 1,11 & 0,69 & 0,57 & 1,42 \\
\hline & $10 \mathrm{~cm}$ & 55,26 & 0,08 & 1,09 & 0,68 & 0,56 & 1,41 \\
\hline & $20 \mathrm{~cm}$ & 61,85 & 0,08 & 0,91 & 0,66 & 0,53 & 1,42 \\
\hline & $30 \mathrm{~cm}$ & 57,42 & 0,06 & 1,02 & 0,72 & 0,58 & 1,43 \\
\hline \multicolumn{2}{|c|}{$\mathrm{DMS}(\mathrm{P})$} & 11,50 & 0,07 & 0,21 & 0,12 & 0,13 & 0,04 \\
\hline \multirow{4}{*}{$\begin{array}{l}\text { CAL } \\
\text { (C) }\end{array}$} & Sem & 53,65 & $0,12 \mathrm{ab}$ & $0,87 \mathrm{bc}$ & $0,71 \mathrm{~b}$ & $0,64 \mathrm{ab}$ & $1,34 \mathrm{~b}$ \\
\hline & SB $40 \%$ & 62,19 & 0,15 a & $1,65 \mathrm{a}$ & $1,09 \mathrm{a}$ & $0,84 \mathrm{a}$ & $1,56 \mathrm{a}$ \\
\hline & SB 50\% & 68,59 & $0,05 \mathrm{bc}$ & $1,09 \mathrm{~b}$ & $0,66 \mathrm{~b}$ & $0,48 \mathrm{bc}$ & $1,47 \mathrm{ab}$ \\
\hline & SB $60 \%$ & 55,66 & $0,04 \mathrm{c}$ & $0,68 \mathrm{c}$ & $0,30 \mathrm{c}$ & $0,30 \mathrm{c}$ & $1,28 \mathrm{~b}$ \\
\hline \multirow{2}{*}{\multicolumn{2}{|c|}{$\begin{array}{c}\mathrm{CV}(\%) \\
\operatorname{DMS}(\mathrm{C})\end{array}$}} & 40 & 74 & 34 & 39 & 37 & 12 \\
\hline & & 15,02 & 0,073 & 0,39 & 0,28 & 0,21 & 0,20 \\
\hline
\end{tabular}

Médias seguidas de mesmas letras nas colunas e mesmo tratamento, não diferem estatisticamente entre si ao nível de 5\% de probabilidade; PROF - Profundidade; CAL - Calcário; CV - Coeficiente de variação. ${ }^{1}$ Transformado em $\sqrt{x^{2}} ;$ Transformados em $\sqrt[7]{x} ;{ }^{3}$ Transformados em $\sqrt[3]{x+1}$.

Fonte: Autores, (2021).

O fosfato reativo possui menor solubilidade quando comparado com outros tipos de adubos fosfatados, devido aos processos para sua obtenção, que passa apenas por processos físicos, enquanto os demais passam por processos químicos com ácido sulfúrico durante sua industrialização, aumentando sua solubilidade em água (Andrade et al, 2019). Em trabalho 
comparando diferentes fontes de fósforo, observou que a utilização de superfosfato simples proporcionou um incremento de 7,1\% em relação ao fosfato reativo (Dias et al, 2015).

O comprimento de raiz também não foi influenciado pelas diferentes doses de calcário, conforme Tabela 2. Não houve interação significativa entre a profundidade de aplicação de fósforo e as doses de calcário, para as variáveis comprimento de raiz, matéria morta, massa de raiz, massa de folha, massa de colmo e na relação folha/colmo. Em relação aos dados quantitativos, o efeito da variável CR não foi significativo (Tabela 2).

A dose de $580 \mathrm{~kg} \cdot \mathrm{ha}^{-1}$, que corrigiu a saturação por bases (SB) para $40 \%$, proporcionou os melhores resultados para as variáveis MM, RAI, FOL, COL e para a relação F/C (Figura 10). Esses resultados evidenciam que para essas variáveis no cultivo de Mombaça, não há necessidade de correção da SB para valores acima de 40\%, visto que com as demais doses os resultados obtidos foram inferiores. Diferindo da SB recomendada para a cultivar, que é classificada como de média exigência, sendo indicada a SB de 50\% (Pereira et al, 2018).

A aplicação de calcário eleva os teores de $\mathrm{Ca}$ e $\mathrm{Mg}$, diminui ou elimina o $\mathrm{Al}$ trocável e aumenta as cargas negativas nesses solos, consequentemente aumentado a disponibilidade de nutrientes, entre eles, o fósforo (Albuquerque, et al., 2003).

Condições de maior acidez (presença de íons $\mathrm{H}^{+}$) são necessárias para aumentar a dissolução. Para os fosfatos naturais de origem apatítica, como é o caso de fosfatos de rocha brasileiros, pode-se esperar uma maior solubilidade em solos ácidos com baixos teores e cálcio disponível, condições típicas das áreas do Cerrado (Goedert et al, 1987).

Figura 1. Regressões para os componentes: massa de matéria morta (A), massa de raiz (B), massa de folha (C), massa de colmo (D) e Relação folha/colmo (E).
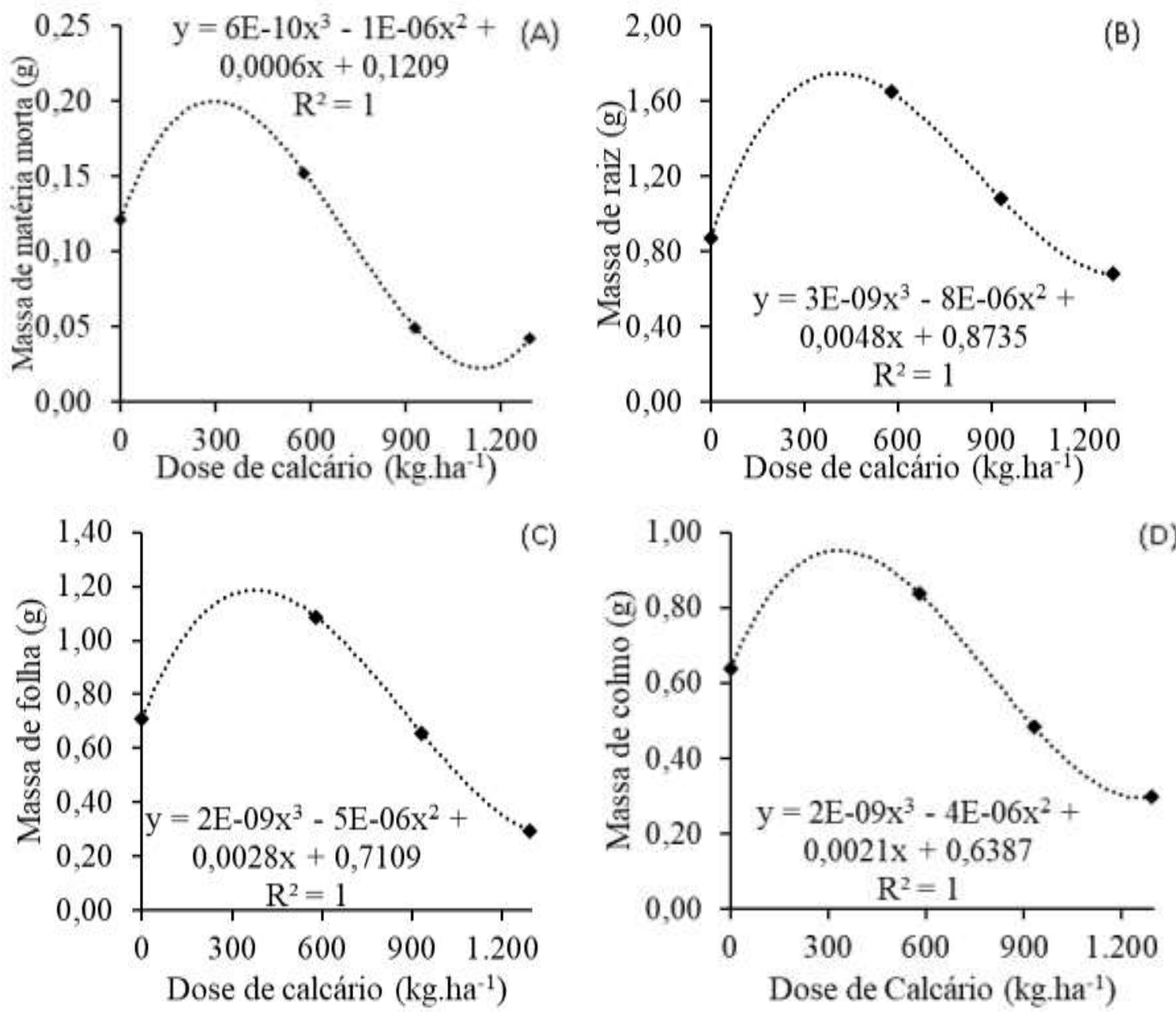


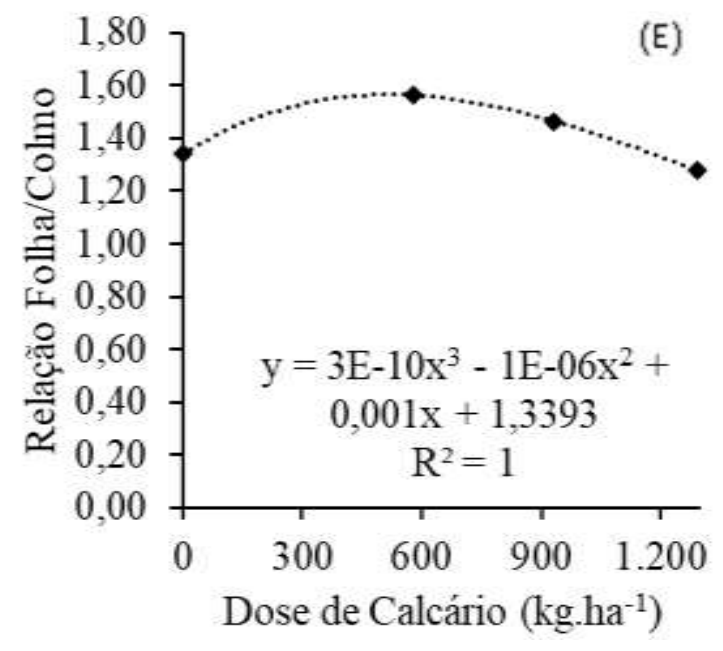

Fonte: Autores (2021).

Houve interação entre as doses de calcário e as profundidades de aplicação de fósforo para as variáveis CF, LF e NP. Sendo que os melhores resultados para CF e LF, no tratamento sem a aplicação de calcário, foram obtidos na ausência de fósforo e quando este foi aplicado em superfície e a $10 \mathrm{~cm}$ de profundidade (Tabelas 3 e 4). Como o pH do solo utilizado no experimento é de 4,45, considerado ácido, e com a presença de $\mathrm{Al}$, provavelmente o fósforo aplicado em maiores profundidades deve ter se fixado ao solo e se tornado indisponível para as plantas (Pereira et al, 2018).

Tabela 3. Desdobramento da interação entre a profundidade de aplicação de fósforo $\mathrm{x}$ as doses de calcário para o comprimento de folha $(\mathrm{CF})$.

\begin{tabular}{|c|c|c|c|c|c|}
\hline \multicolumn{6}{|c|}{ Comprimento de folha } \\
\hline & & & CAL $(\mathrm{kg}$ & \multirow[b]{2}{*}{930} & \multirow[b]{2}{*}{1.290} \\
\hline & & 0 & 580 & & \\
\hline \multirow{5}{*}{ PROF } & Sem & 4,65 A a & $4,73 \mathrm{~A}$ & $3,73 \mathrm{AB}$ & $2,48 \mathrm{~B}$ \\
\hline & Sup & $4,15 \mathrm{~A}$ ab & $4,06 \mathrm{~A}$ & $4,01 \mathrm{~A}$ & $2,61 \mathrm{~B}$ \\
\hline & $10 \mathrm{~cm}$ & $3,41 \mathrm{AB} a b c$ & $4.19 \mathrm{~A}$ & $3,95 \mathrm{~A}$ & $2,44 \mathrm{~B}$ \\
\hline & $20 \mathrm{~cm}$ & $1,96 \mathrm{~B} \mathrm{c}$ & $4,99 \mathrm{~A}$ & $3,08 \mathrm{~B}$ & $2,76 \mathrm{~B}$ \\
\hline & $30 \mathrm{~cm}$ & $2,80 \mathrm{~B}$ bc & $5,01 \mathrm{~A}$ & 3,64 B & $2,43 \mathrm{~B}$ \\
\hline & (\%) & 17 & & & \\
\hline & PROF) & $\begin{array}{l}1,47 \\
1,99\end{array}$ & & & \\
\hline & CAL) & 1,99 & & & \\
\hline
\end{tabular}

Médias seguidas de mesmas letras minúsculas nas colunas e maiúsculas nas linhas, não diferem estatisticamente entre si ao nível de 5\% de probabilidade; PROF - Profundidade; CAL - Calcário; CV - Coeficiente de variação. Médias transformadas em $\sqrt{x}$. Fonte: Autores (2021). 
Tabela4. Desdobramento da interação entre a profundidade de aplicação de fósforo x as doses de calcário para a largura de folha (LF).

\begin{tabular}{|c|c|c|c|c|c|}
\hline \multicolumn{6}{|c|}{ Largura de folha } \\
\hline & & \multicolumn{4}{|c|}{$\operatorname{CAL}\left(\mathrm{kg} \mathrm{ha}^{-1}\right)$} \\
\hline & & & 580 & 930 & 1.290 \\
\hline \multirow{5}{*}{ PROF } & Sem & $1,45 \mathrm{~A} \quad \mathrm{a}$ & $1,47 \mathrm{~A}$ & $1,45 \mathrm{AB}$ & $0,43 \mathrm{~B}$ \\
\hline & Sup & $1,33 \mathrm{~A} \quad \mathrm{ab}$ & $1,16 \mathrm{~A}$ & $1,08 \mathrm{~A}$ & $0,42 \mathrm{~B}$ \\
\hline & $10 \mathrm{~cm}$ & $0,91 \mathrm{AB} a b c$ & $1,29 \mathrm{~A}$ & $1,13 \mathrm{~A}$ & $0,41 \mathrm{~B}$ \\
\hline & $20 \mathrm{~cm}$ & $0,28 \mathrm{~B} \quad \mathrm{c}$ & $1,58 \mathrm{~A}$ & $0,81 \mathrm{~B}$ & $0,42 \mathrm{~B}$ \\
\hline & $30 \mathrm{~cm}$ & $0,61 \mathrm{~B} \quad \mathrm{bc}$ & $1,60 \mathrm{~A}$ & $1,01 \mathrm{AB}$ & $0,43 \mathrm{~B}$ \\
\hline \multicolumn{2}{|c|}{$\mathrm{CV}(\%)$} & \multicolumn{2}{|c|}{31} & & \\
\hline \multicolumn{2}{|c|}{ DMS (PROF) } & \multicolumn{2}{|c|}{0,82} & & \\
\hline \multicolumn{2}{|c|}{ DMS (CAL) } & \multicolumn{2}{|c|}{1,03} & & \\
\hline
\end{tabular}

Médias seguidas de mesmas letras minúsculas nas colunas e maiúsculas nas linhas, não diferem estatisticamente entre si ao nível de 5\% de probabilidade; PROF - Profundidade; CAL - Calcário; CV - Coeficiente de variação.

Fonte: Autores (2021).

Na ausência de calagem o NP foi maior sem fósforo e quando este foi aplicado em superfície e nas profundidades de 10 e $30 \mathrm{~cm}$ (Tabela 5). Pereira et al, (2018) ressalta que na presença Al, que é o caso do solo utilizado, formam-se compostos insolúveis que tornam o fósforo não disponível para as plantas, os autores ainda estimam que de todo fósforo aplicado no solo, a planta aproveita apenas de 15 a $25 \%$.

Tabela 5. Desdobramento da interação entre a profundidade de aplicação de fósforo x as doses de calcário para o número de perfilhos (NP).

Número de Perfilhos

\begin{tabular}{|c|c|c|c|c|c|}
\hline & & \multicolumn{4}{|c|}{ CAL $\left(\mathrm{kg} \mathrm{ha}^{-1}\right)$} \\
\hline & & 0 & 580 & 930 & 1.290 \\
\hline \multirow{5}{*}{ PROF } & Sem & $3,00 \mathrm{~A} \mathrm{a}$ & $2,33 \mathrm{~A}$ & $1,33 \mathrm{AB}$ & $0,00 \mathrm{~B}$ \\
\hline & Sup & $2,67 \mathrm{~A} \mathrm{a}$ & $2,33 \mathrm{AB}$ & $1,67 \mathrm{AB}$ & $0,33 \mathrm{~B}$ \\
\hline & $10 \mathrm{~cm}$ & $1,00 \mathrm{~A} \mathrm{a}$ & $2,22 \mathrm{~A}$ & $2,11 \mathrm{~A}$ & $0,22 \mathrm{~A}$ \\
\hline & $20 \mathrm{~cm}$ & $0,00 \mathrm{~B} \quad \mathrm{~b}$ & $3,11 \mathrm{~A}$ & $0,22 \mathrm{~B}$ & $0,33 \mathrm{~B}$ \\
\hline & $30 \mathrm{~cm}$ & $0,67 \mathrm{~B} \mathrm{ab}$ & $3,78 \mathrm{~A}$ & $1,22 \mathrm{~B}$ & $0,33 \mathrm{~B}$ \\
\hline \multicolumn{2}{|c|}{$\mathrm{CV}(\%)$} & 66 & & & \\
\hline \multicolumn{2}{|c|}{ DMS (PROF) } & 2,40 & & & \\
\hline \multicolumn{2}{|c|}{ DMS (CAL) } & 2,36 & & & \\
\hline
\end{tabular}

Médias seguidas de mesmas letras minúsculas nas colunas e maiúsculas nas linhas, não diferem estatisticamente entre si ao nível de 5\% de probabilidade; PROF - Profundidade; CAL - Calcário; CV - Coeficiente de variação.

Fonte: Autores (2021).

Quando realizada a calagem do solo, elevando a SB para 40, 50 e $60 \%$ a profundidade de aplicação de fósforo não influenciou as variáveis CF, LF e o NP. Esses resultados foram obtidos em função da correção da acidez do solo que potencializa a disponibilização de nutrientes para as plantas. De acordo com Pereira et al, (2018) quando ácidos os solos não 
permitem que boa parte dos nutrientes estejam disponíveis para as plantas, o que resulta em baixo potencial de crescimento do pasto.

Os resultados da variável comprimento de folhas se ajustaram a regressão quadrática para as diferentes doses de calcário, sem a aplicação de fósforo, quando este foi aplicado em superfície e nas profundidades de 10 e $30 \mathrm{~cm}$ (Figura 2). Enquanto, o tratamento com fósforo aplicado a $20 \mathrm{~cm}$ de profundidade se ajustou a regressão cúbica.

Efeito inverso das doses de calcário foi observado no comprimento de folhas de Mombaça, enquanto o gesso proporcionou incrementos. Os maiores valores do $\mathrm{pH}$ do solo, provocado pelas maiores doses de calcário, podem ter reduzido a disponibilidade de fósforo, já no caso do gesso, as doses mais elevadas podem ter provocado reações químicas nas quais o sulfato oriundo do gesso, poderia ocupar sítios de adsorção de fósforo, fazendo com que esse elemento permanecesse mais tempo na forma lábil (Portz et al, 2015).

Figura 2. Regressões para os componentes comprimento de folha sem fósforo (A), fósforo em superfície (B), fósforo a $10 \mathrm{~cm}$ (C), fósforo a $20 \mathrm{~cm}$ (D) e fósforo a $30 \mathrm{~cm}$ (E).
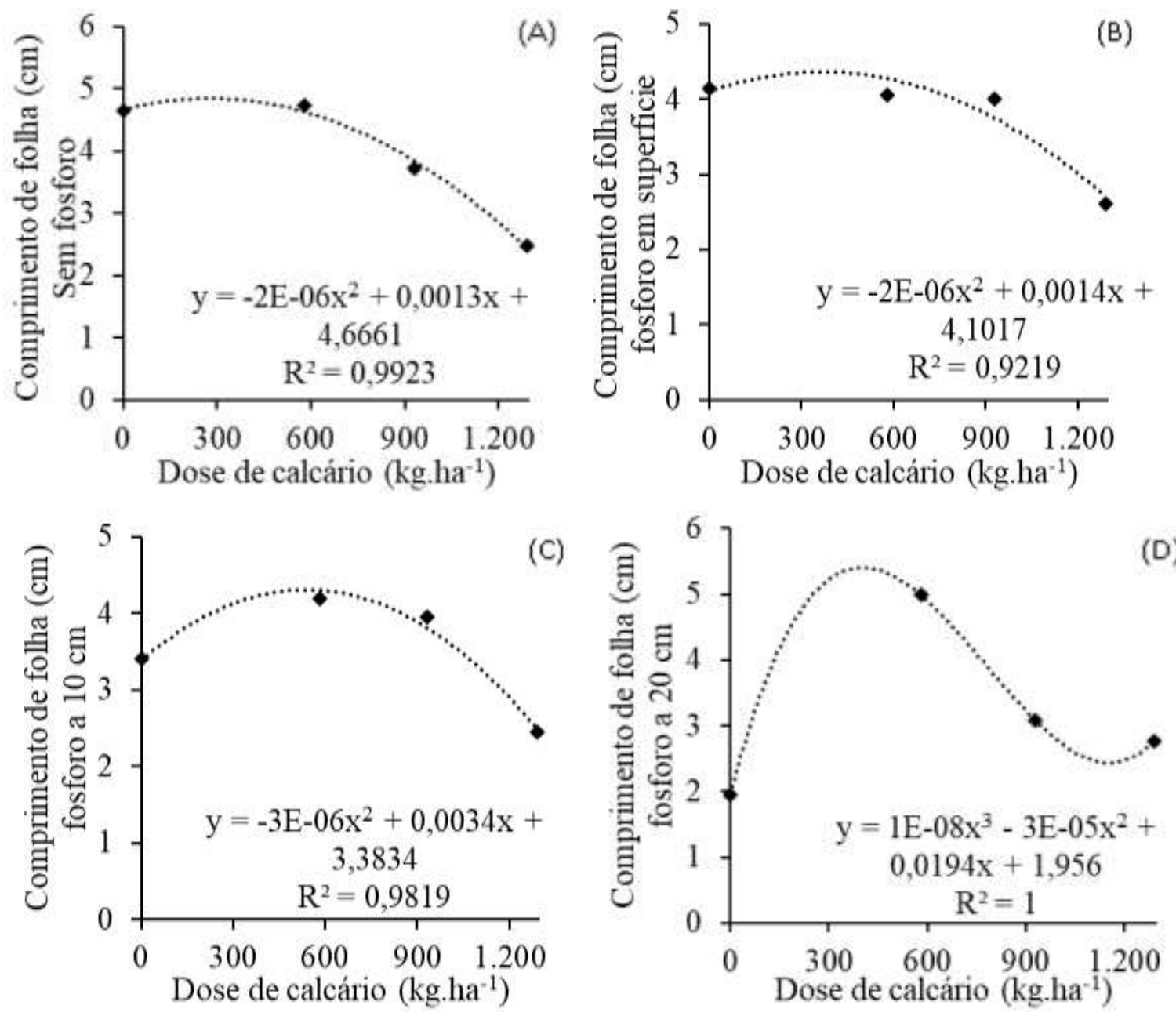


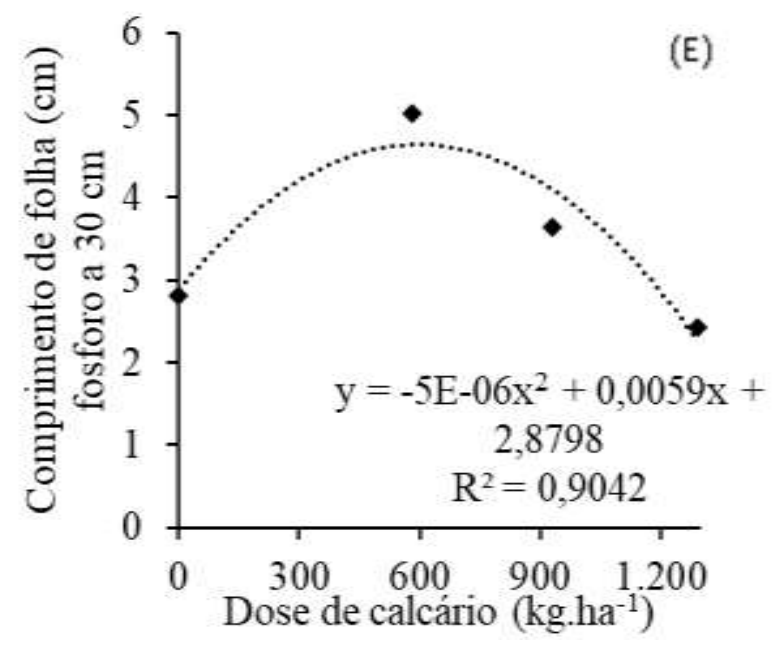

Fonte: Autores (2021).

Para a largura de folhas os resultados se ajustaram a regressão quadrática quando o fósforo foi aplicado a 10 e $30 \mathrm{~cm}$ de profundidade e na ausência deste, já quando este foi aplicado na superfície os resultados se ajustaram a uma regressão linear, e quando aplicado a $20 \mathrm{~cm}$ de profundidade os resultados apresentaram regressão quadrática (Figura 3).

Figura 3. Regressões para os componentes largura de folha sem fósforo (A), fósforo em superfície (B), fósforo a $10 \mathrm{~cm}(\mathrm{C})$, fósforo a $20 \mathrm{~cm}$ (D) e fósforo a $30 \mathrm{~cm}$ (E).
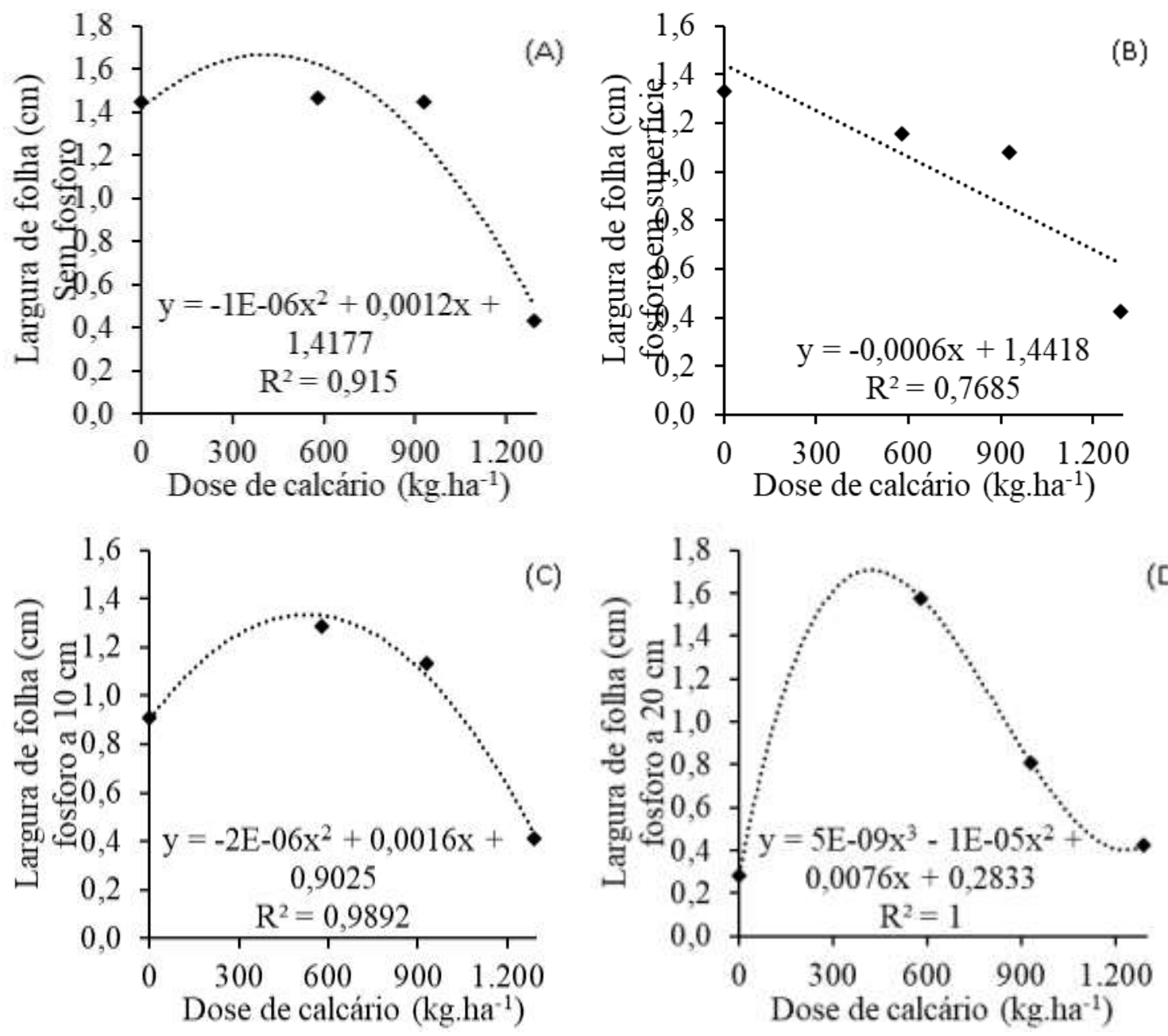


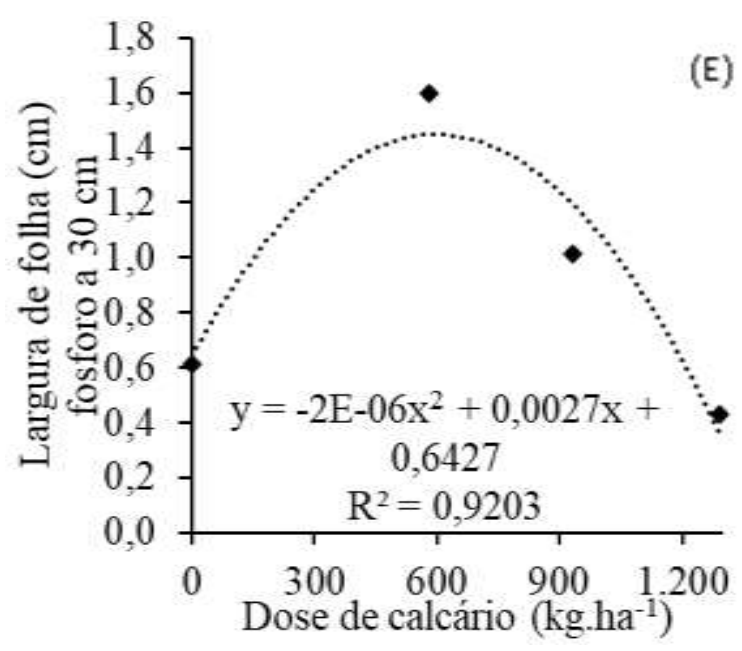

Fonte: Autores (2021).

Com exceção da adubação em que o fósforo foi aplicado em superfície, nos demais tratamentos os melhores resultados para a largura de folhas foram obtidos quando a saturação de bases foi corrigida para $40 \%$. Tal resultado pode estar associado a maior disponibilização de fósforo para as plantas nessa condição de saturação. Souza et al. (2006) em estudo com níveis de saturação, observou que o maior teor de fósforo foi obtido com 40 \% de SB.

Já os resultados do número de perfilhos se ajustaram a regressão linear decrescente quando o fósforo foi aplicado na superfície e na ausência de adubação. Já para o fósforo aplicado a $10 \mathrm{~cm}$ de profundidade, os resultados se ajustaram a regressão quadrática. E nas demais profundidades os resultados apresentaram regressão cúbica (Figura 4). 
Figura 4. Regressões para os componentes número de perfilhos sem fósforo (A), fósforo em superfície (B), fósforo a $10 \mathrm{~cm}$ (C), fósforo a $20 \mathrm{~cm}$ (D) e fósforo a $30 \mathrm{~cm}(\mathrm{E})$.
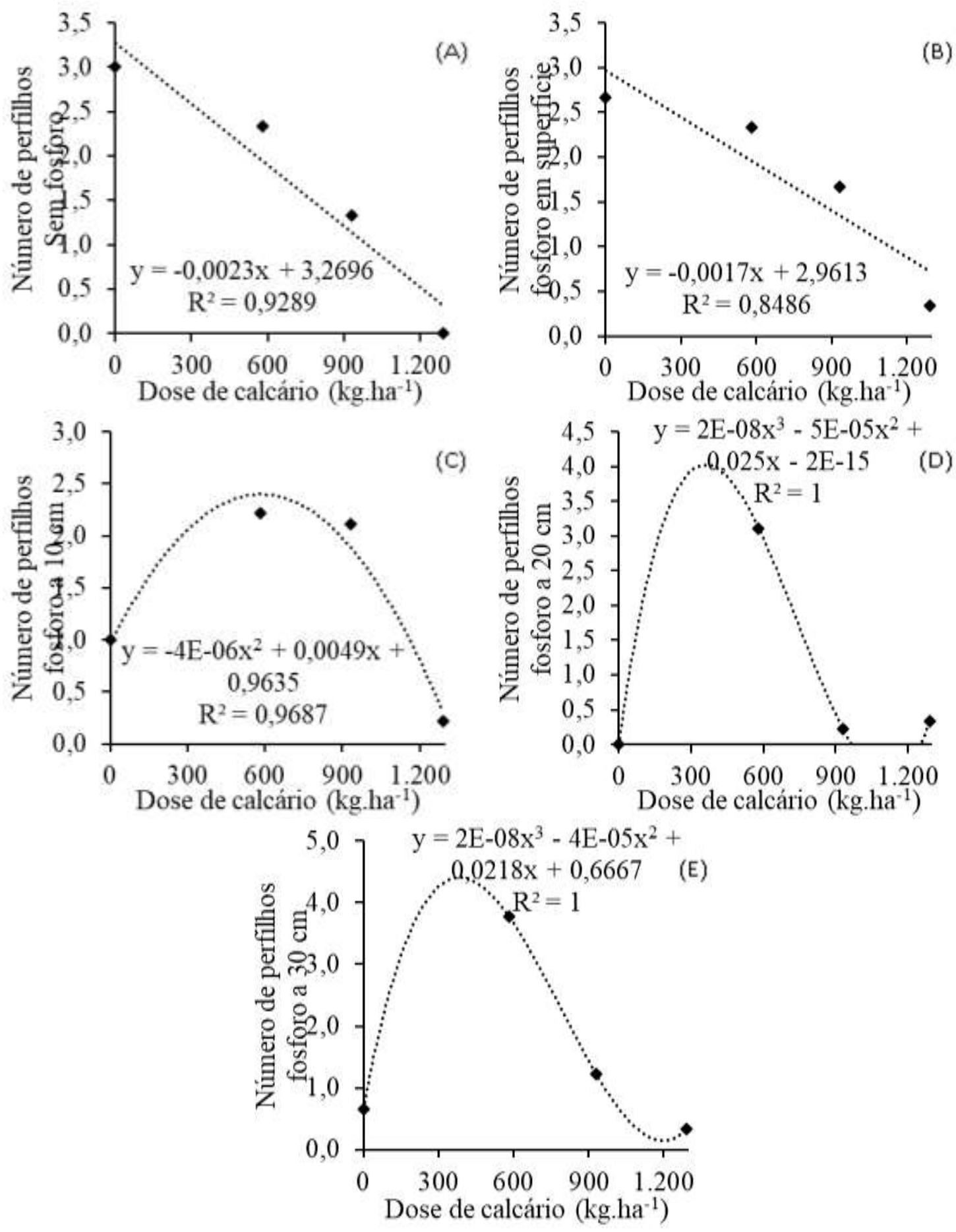

Fonte: Autores (2021).

Nas regressões lineares os melhores resultados do NP foram obtidos sem a aplicação de calcário, seguido da correção da saturação para 40\%. Quando o fósforo foi aplicado em profundidade, independente do ajuste de regressão os melhores resultados desta variável foram obtidos com a correção da SB para $40 \%$, sendo que nas demais saturações o número de perfilhos obtidos foram próximos a zero, ou seja, ausência de perfilhamento. 
Já Florentino et al. (2019), observaram que o NP aumenta em função das doses de fósforo. Deste modo, os resultados obtidos no presente trabalho podem estar relacionados ao relatado por Portz et al. (2015), que observaram redução na disponibilidade de fósforo, em função dos maiores valores do pH do solo, obtidos com as maiores doses de calcário.

A aplicação dos fertilizantes fosfatados em função de doses proporcionou uma maior produtividade de massa seca da parte aérea do capim Panicum maximum cv. Mombaça. A adubação com as diferentes fontes e doses de fósforo influenciaram de forma significativa a produção da forrageira Panicum maximum cv. Mombaça, aumentando a altura do capim, número de perfilhos, massa verde, massa seca da parte aérea. A maior produção do capim foi nas doses de 280, 215, 239 e $207 \mathrm{~kg}$.ha-1 de $\mathrm{P}_{2} \mathrm{O}_{5}$ para as fontes UFT Fértil, Fosfato Natural, Basifós e Superfosfato Simples respectivamente (Carneiro et al, 2017).

Trabalhando com diferentes fontes de P, Cecato et al. (2008), observaram que fontes com maior solubilidade como Superfosfato simples e posteriormente o Yoorin, proporcionaram um maior incremento foliar e posteriormente maior quantidade de perfilhos. Ainda destacam que o fósforo tem maior influência no perfilhamento do que no alongamento foliar em gramíneas, pois desempenha papel importante nas características morfológicas e produção desse capim.

Em trabalho com diferentes fontes de fósforo (farinha de ossos, fosfato de Arad e superfosfato simples, na dose de 70 $\mathrm{kg}$ de $\mathrm{P}_{2} \mathrm{O}_{5}$ ha $^{-1}$ ) também observaram que a adubação fosfatada promoveu maior perfilhamento e maior produtividade do capim mombaça corroborando com os resultados do trabalho citado acima (Oliveira et al, 2012).

A fonte de fósforo utilizada (fosfato natural de Bonito) supriu a demanda da forrageira em relação ao manejo adotado, podendo assim ser empregada em áreas de pastagens objetivando retorno financeiro e uma agricultura sustentável.

\section{Considerações Finais}

A correção da saturação de bases para $40 \%$ proporciona os melhores resultados de CF, LF e NP, independente da profundidade de aplicação de fósforo.

\section{Agradecimentos}

Os autores agradecem a Empresa de Desenvolvimento em Mineração e Participações Ltda - EDEM pela cessão do insumo para o projeto científico.

\section{Referências}

Andrade, R. A., Porto, M. O., Cavali, J., Ferreira, E., Bergamin, A. C., Souza, F. R. \& Aguiar, I. S. (2019). Azospirillum brasilense e fosfato natural reativo no estabelecimento de forrageira tropical. Revista de Ciências Agrárias. 42. 146-154.

Araújo, L. M. B., Andrade, A. C., Rodrigues, B. H. N., Santos, F. J. S., Magalhães, J. A., Rodrigues, R. C. \& Oliveira, I. V. L. (2019). Produtividade do capim mombaça sob diferentes idades de rebrotação no norte do Piauí. 16. 1. Nucleus. https://ainfo.cnptia.embrapa.br/digita 1/bitstream/item/197220/1/PRODUTIVIDADE-DO-CAPIM-MOMBACA-SOB-DIFERENTES-IDADES.pdf.

Carneiro, J. S. S., Silva, P. S. S., Santos, A. C. M., Freitas, G. A. \& Silva, R. R. (2017). Resposta do capim mombaça sob efeito de fontes e doses de fósforo na adubação de formação. Journal of Bioenergy and Food Science. .4.1, 12-25. 2017. https://doi.org/10.18067/jbfs.v4i1.117.

Cecato, U., Skrobot, V. D., Fakir, G. R., Branco, A. F., Galbeiro, S. \& Gomes, J. A. N. (2008). Perfilhamento e características estruturais do capim mombaça, adubado com fontes de fósforo, em pastejo. Acta Scientiarum Animal Scienses. 30. 1. Universidade Estadual de Maringá. p 1-7.

Dias, D. G., Pegoraro, R. F., Alves, D. D., Porto, E. M. V., Santos Neto, J. A. \& Aspiazú, I. (2015). Produção do capim Piatã submetido a diferentes fontes de fósforo. Revista Brasileira de Engenharia Agrícola Ambiental. 19(4)330-335.

Dias-filho, M. B. (2016). Uso de pastagens para a produção de bovinos de corte no Brasil: Passado, Presente e Futuro. Documentos 418 . Embrapa Amazônia Oriental. Belém: PA. 42.

Duda, G. P. \& Salviano, A. M. (2007). Alterações na fertilidade de um latossolo devido à prática da calagem recomendada pelo método de incubação. Revista Científica Rural. 12. 1. 28-35.

EMBRAPA. (2018). Sistema brasileiro de classificação de solo. (5a ed.). 353. 
Research, Society and Development, v. 10, n. 13, e361101313794, 2021

(CC BY 4.0) | ISSN 2525-3409 | DOI: http://dx.doi.org/10.33448/rsd-v10i13.13794

Ferreira, E. (2014). Teste de comparações múltiplas bootstrap no pacote ExpDes para DBC e DQL. Revista da Estatística da Universidade Federal de Ouro Preto. 3. 3. 729-733.

Florentino, L. S., Santos, R. M., Oliveira, A. A., Landes, T. S. \& Picazevicz, A. A. C. (2019). Crescimento do capim Panicum maximum cv. "Mombaça" em resposta a adubação fosfatada na Amazônia Ocidental. Enciclopédia Biosfera. 16. 30. 153.

Goedert, W. J., Sousa, D. M. C. \& Scolari, D. D. C. (1987). Critérios para recomendação de calagem e adubação. Circular Técnica n. 25. Planaltina:DF. Embrapa Centro de Pesquisa Agropecuária dos Cerrados. 55.

Lopes, A. S. (1998). Manual Internacional de Fertilidade do Solo. (2a ed.). Potafos. 177.

Oliveira, S. B., Caione, G., Camargo, M. F., Oliveira, A. N. B. \& Santana, L. (2012). Fontes de fósforo no estabelecimento e produtividade de forrageiras na região de alta Floresta - MT. Global Science And Technology, 05. 01. 01 - 10.

Pereira, L. E. T., Nishida, N. T., Carvalho, L. R. \& Herling, V. R. (2018). Recomendações para correção e adubação de pastagens tropicais. FZEA/USP. 01. 56.

Portz, A., Martins, C. A. C., Silva, G. L. S. \& Zonta, E. (2015). Crescimento de capim Mombaça com aplicação de calcário e gesso em um Argissolo sul fluminense. XXXV Congresso Brasileiro de Ciência do Solo. 1. 4.

Raij, B V., Cantarella, H.; Quaggio, J. A. \& Furlani, A. M. C. (1997). Recomendações de adubação e calagem para o estado de São Paulo Boletim técnico 100. 2. Edição. Campinas. Instituto Agronômico Fundação IAC. 285.

Ramos G. M., Italiano, E. C., Leite, G. G., Melo, F. B., Ribeiro, V. Q. (1993). Doses de fósforo na produção de gramíneas forrageiras em solos ácidos e de baixa fertilidade da região meio-norte do Brasil. Pasturas tropicales. 9(3), 24-27.

Rossa, U. B. (2006). Estimativa de calagem pelo método SMP para alguns solos do Paraná. (2006). Dissertação (Mestrado). Universidade Federal do Paraná, Curitiba-PR

Souza, D. M. G. de, Vilela, L., Lobato, E. \& Soares, W. V. (2001). Uso de gesso, calcário e adubos para pastagens no cerrado. Circular técnica 12. Embrapa Cerrados. Planaltina: DF. 22.

Souza, R. M., Pinto, J. C., Evangelista, A. R. \& Couto Filho, C. C. C. (2006). Formas de aplicação de calcário nos teores de minerais da forragem do capim tanzânia. Ciência Agrotec. 30. 4. 752-758.

R Core Team. (2019). R: A Language and Environment for Statistical Computing. R Foundation for StatisticalComputing. Vienna, Austria. URL https:/www.R-project.org/.

Tennant, D. A. (1975). Test of a mobifiedline intersect methodo stimatin groot length. Journal of Ecology. 63. 3, 995-1001.

Wickham, H. (2010). ggplot2: gráficos elegantes para análise de dados. J StatSoftw. 35, 1. 65-88. 